\title{
Lightweight in Automotive Components by Forming Technology
}

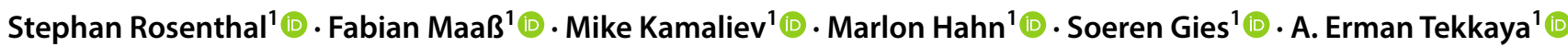

Received: 28 February 2020 / Accepted: 18 June 2020 / Published online: 31 July 2020

(c) The Author(s) 2020

\begin{abstract}
Lightweight design is one of the current key drivers to reduce the energy consumption of vehicles. Design methodologies for lightweight components, strategies utilizing materials with favorable specific properties and hybrid materials are used to increase the performance of parts for automotive applications. In this paper, various forming processes to produce light parts are described. Material lightweight design is discussed, covering the manufacturing processes to produce hybrid components like fiber-metal, polymer-metal and metal-metal composites, which can be used in subsequent deep drawing or combined forming processes. Approaches to increasing the specific strength and stiffness with thermomechanical forming processes as well as the in situ control of the microstructure of such components are presented. Structure lightweight design discusses possibilities to plastically form high-strength or high-performance materials like magnesium or titanium in sheet, profile and tube forming operations. To join those materials and/or dissimilar materials, new joining by forming technologies are shown. To economically produce lightweight parts with gears or functional elements, incremental sheet-bulk metal forming is presented. As an important part property, the damage evolution during the forming operations will be discussed to enable even lighter parts through a more reliable design. New methods for predicting and tailoring the mechanical properties like strength and residual stresses will be shown. The possibilities of system lightweight design with forming technologies are presented. A combination of additive manufacturing and forming to produce highly complex parts with integrated functions will be shown. The integration of functions by a hot extrusion process for the manufacturing of shape memory alloys is presented. An in-depth understanding of the newly developed processes, methodologies and effects allows for a more accurate dimensioning of components. This facilitates a reduction in the total mass and an increasing performance of vehicle components.
\end{abstract}

Keywords Forming technology $\cdot$ Lightweight design $\cdot$ Energy efficiency $\cdot$ Manufacturing processes

\section{Introduction}

Lightweight design is a key driver to reduce $\mathrm{CO}_{2}$ emissions in transportation and automotive industries. The increasing demand for electric and hybrid powered cars requires new concepts and development of lightweight technologies and processes to produce light parts and automotive components. Lightweighting is a constructional philosophy aiming for maximal weight savings of components and modules. Three different strategies of lightweight design are used to structure the paper.

Stephan Rosenthal

Stephan.Rosenthal@iul.tu-dortmund.de

1 Institute of Forming Technology and Lightweight Components (IUL), TU Dortmund University, 44227 Dortmund, Germany

\section{(1) Material lightweight design}

This design strategy utilizes the benefits of the material itself. Depending on the density and the material properties, different materials reach various levels of strength and/or stiffness. Material lightweight design can be done by the use of a single material with a high specific property or by a combination of materials to utilize the best of different materials which are combined to a composite or hybrid. Material lightweight design is a subset of structure lightweight design.

\section{(2) Structure lightweight design}

It is a concept to develop and design parts by topology, shape and parameter optimization. The aim is to change the shape and form to reduce the weight while the stiffness and/ or strength is increased or kept constant. The combination of 
structures to an assembly can lead to a light system; therefore, structure lightweight design is a subset of system lightweight design.

\section{(3) System lightweight design}

This means the combination of parts to a system that is lighter than a reference system. The system always consists of components that are combined to an assembly, such as a car or modules of a car like the structural cage. To produce a light system, the components of the system need to be light by themselves. The integration of functions or the reduction in parts by integrating them into a new component is system lightweight design as well. To reach the aim of light parts, the next level of lightweight design is a subset of system lightweight design.

All aforementioned lightweight strategies are aiming for a maximum weight reduction. A critical aspect is the choice of the reference system which is used for the calculation of the weight reduction. Hahn et al. [1] developed a new concept to evaluate the design and level of lightweight - the true lightweight degree. This new factor overcomes the current industrial practice, where a new component is typically associated with lightweight design, if it is lighter than the previous version of the part. The true lightweight degree ensures additivity along the development chain, and it enables to predict the mass-minimizing material choice for a stiffness-oriented component design [1].
Innovative processes and material concepts are required for the production of lightweight components. The majority of vehicle components are manufactured using forming technologies. Figure 1 illustratively shows various vehicle components that can be manufactured using innovative forming processes. The figure is set up to navigate to the specific sections in this paper to give more information about each mentioned process. The corresponding parts that can be manufactured with the later presented processes are pointed out in Fig. 1 as well.

Electromobility is evolving rapidly during recent years and is getting a more important role in future manufacturing facilities; Fig. 2 shows potential use cases for forming applications in this comparatively new vehicle type. Comparing combustion vehicles and electric vehicles as shown in Figs. 1 and 2, the differences are clearly visible. Nearly the whole drive train assembly is substituted by electrical components such as the battery assembly and the electrical motors. Specially, the battery housing plays a key role in crash safetyness [2] and needs to be protected in case of an accident.

A part property that theoretically stands above all manufacturing processes and material concepts is the damage evolution during manufacturing which is discussed in Sect. 3.4. Damage is an important property when dealing with lightweight design and is defined as the void evolution inside a part during or after a forming operation. It enables the knowledge of process-induced void growth, which weakens

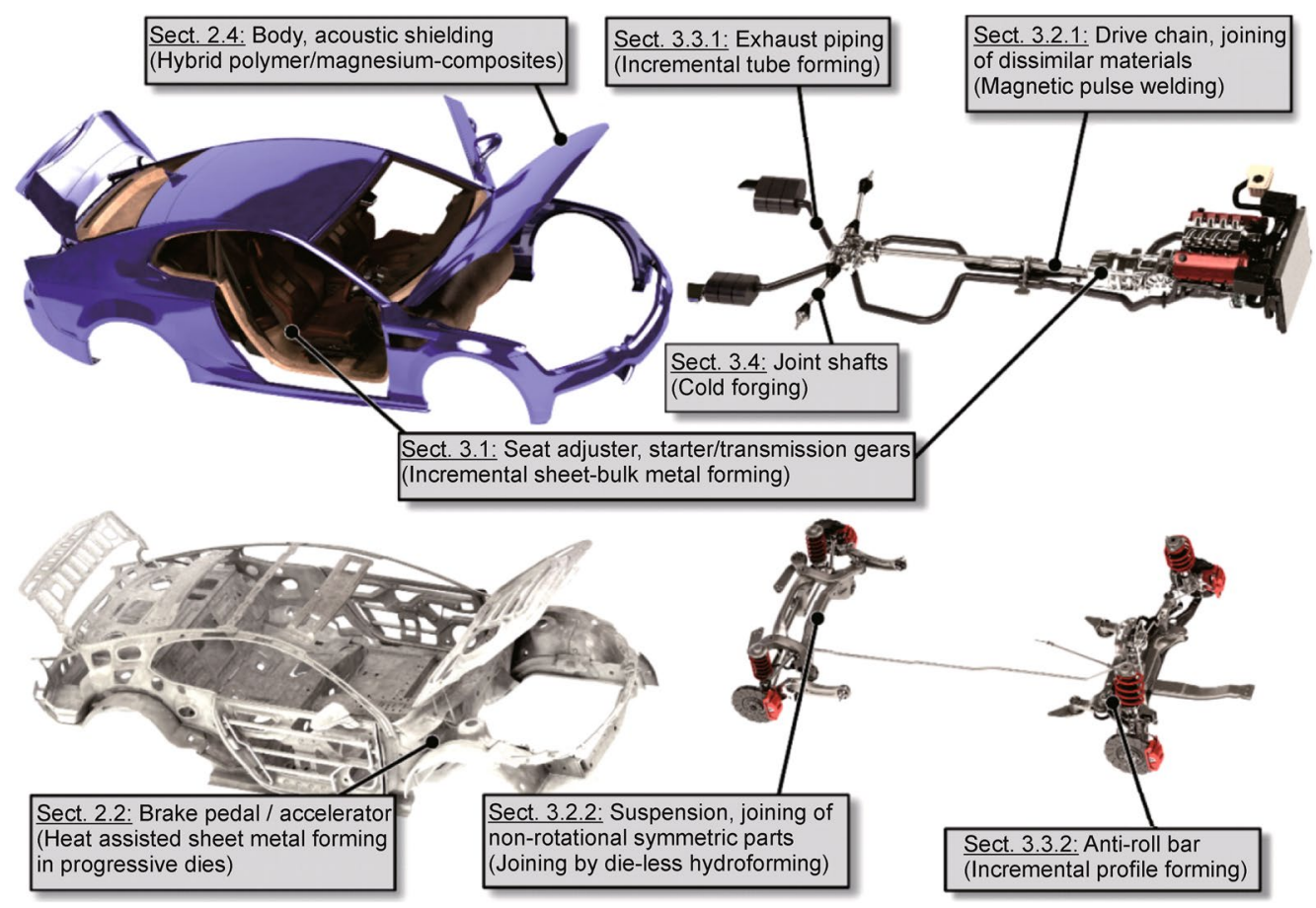

Fig. 1 Processes that can be used to manufacture lightweight automotive components 
Fig. 2 Processes that can be used to manufacture lightweight automotive components for electric vehicles

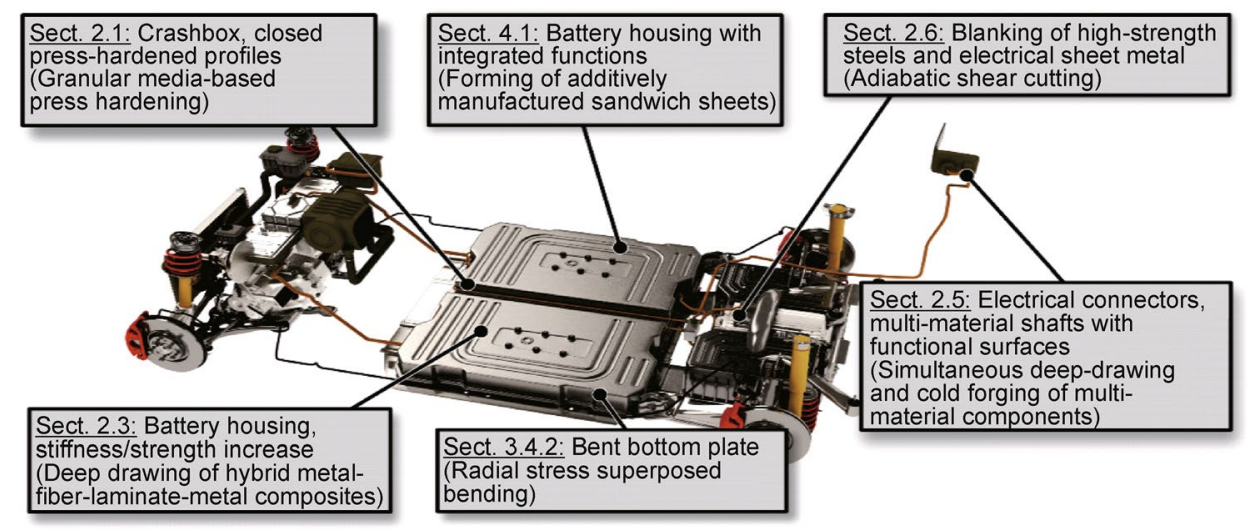

the parts performance. A deep understanding of this effect helps to properly rate and design a part for a specific load case without the danger of over-engineering and using more material than necessary.

This paper focuses on processes and material concepts to produce light parts for the automotive industry using forming technologies.

\section{Material Lightweight Design by Forming Technology}

Forming processes focusing on material lightweight design usually replace the primary material by another material with better specific weight characteristics. For this, highstrength steels or light metals are used, whereas the geometry of the workpiece has no significant influence on the weight [3].

\subsection{Granular Media-Based Press Hardening of Closed Profiles}

Lightweight design and crash safety are conflicting aims in automotive engineering that demand suitable forming processes. Hot stamping is an established forming process used in the automobile industry to meet the demands for safety and lightweight design combined with an appropriate setting of mechanical properties [4]. Almost 30\% of modern car body parts are processed by hot stamping, and the amount is continuously rising [5]. The key for manufacturing highstrength and complex-shaped sheet metal components is the combined forming and heat treatment. Boron micro-alloyed steel sheets like $22 \mathrm{MnB} 5$ are heated to austenitization temperature before they are formed and quenched in the closed tool simultaneously. These press-hardened steels can reach strength of up to $1500 \mathrm{MPa}$ [6].
The process characteristics of hot stamping can also be extended to hollow profiles with high strength and high stiffness. A typical forming process to form closed profiles is hydroforming, but in combination with high temperatures a special forming medium is needed. Besides gas, shapeless solids like granular materials are suitable alternatives [7].

Another challenging aspect is that a particle-based medium does not behave hydrostatically like a fluid or gaseous medium. The force is distributed along force chains, which is depending on punch geometry and friction conditions inside the medium as well as between the medium and the tube. The combination of low inter-particle friction, low Poisson's ratio and large tube diameters is optimal conditions for effective force transmission and thus for a successful forming result [8].

Different granular materials have varying characteristics regarding force transmission. Compared with zirconia bead, quartz sand transfers higher radial pressure under the same loading condition. On the other hand, it generates higher friction during interaction with the tube wall. Thus, the radial pressure decreases with increasing distance to the loading zone [7].

Granular media can also be used as passive medium [9]. It is placed inside the profile, and a punch forms the tube with direct contact on the outer tube surface. Unlike the active use of the medium, there is no contact between punch and the granular medium. The pressure loss due to interfacial friction between tube and granular medium is reduced, and the pressure generation inside the medium is optimized. Figure 3 shows different kinds of parts that can be formed by active and passive granular media-based press hardening.

The described process enables the manufacturing of parts with high strength due to the press-hardened material and by combination gives the part a very unique shape to meet the stiffness requirements if needed. Therefore, these parts offer great potentials for lightweight designs, 


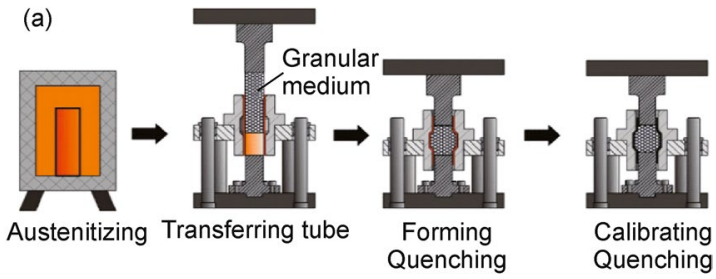

(b)
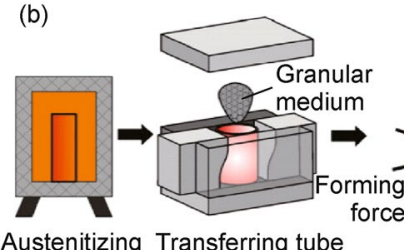

(c)

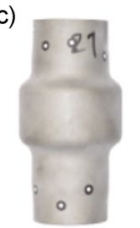

(d)

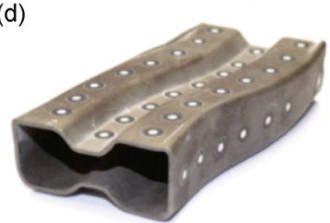

Fig. 3 Granular media-based press hardening processes a with active granular medium; b with passive granular medium; c with active medium on the inside; $\mathbf{d}$ passive medium on the inside (taken from [8])

e.g., in safety elements in crashboxes, in battery housings of electric vehicles, respectively, like shown in Fig. 2.

\subsection{Sheet Metal Forming in Progressive Dies Assisted by Rapid Induction Heating}

Using hot stamping in a multi-stage progressive die, the high demands on the tailor-made steel products should be realized at low costs and for large quantities.

Löbbe et al. [10] developed a new rapid multi-step sheet metal forming process to manufacture small- and mediumsized parts, as shown in Fig. 4. The process is based on in situ inductive heating and progressive or transfer tools and was invented by Becker et al. [11]. In order to compensate for large springback and enhance the forming limit, a closedloop control has been implemented. A measurement of the springback angle is used to adjust the temperature of the workpiece till a stable process is generated. By separating the forming and cooling over several stages, a higher stroke rate, proportional to the number of stages, can be achieved until the limit of the heating utility or the whole process facility is reached.

The limiting factor in this process design is usually the inductive heating, which allows heating rates up to $200 \mathrm{~K} / \mathrm{s}$ [12] which makes it ideal for the rapid multi-step forming process. Rapid hot forming based on high power and process-integrated heating methods is an approach to reaching outstanding mechanical properties and a more flexible design by forcing dynamic phase transformation effects and locally adapted thermal cycles.

This heat-assisted rapid forming process offers the possibility to produce high amounts of parts to be used inside or to protect the passenger cell. A possible application is the manufacturing of lightweight brake or accelerator pedals with a specific shape to stiffen those parts.

\subsection{Deep Drawing of Hybrid Fiber-Metal Laminate Composites}

The automotive industry started to use metal-polymer-metal products for body-in-white components in the 1980 s to decrease the total weight, driven by the increasing oil prices and their beneficial specific strength to weight properties [13]. The production of complex-shaped lightweight components from composites was impeded by missing manufacturing processes. Mennecart et al. [14] analyzed different strategies to form fiber-metal laminates by deep drawing and resin transfer molding (RTM), and deep drawing combined with wet pressing. The benefit
Fig. 4 Process principle of heatassisted sheet metal forming in progressive dies [10]

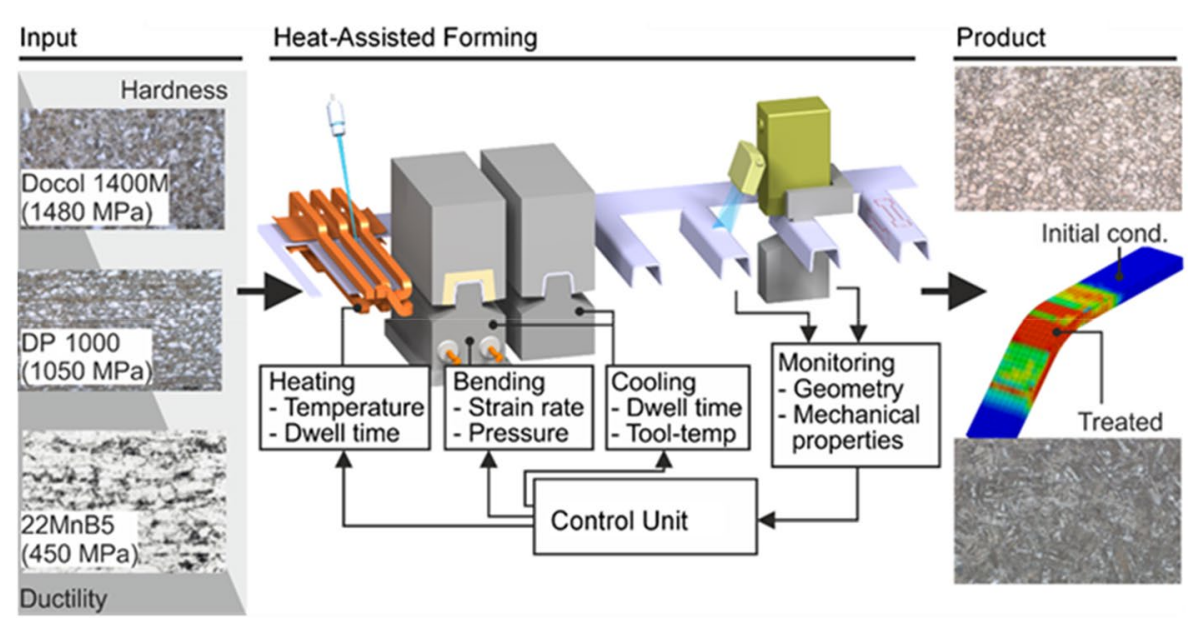


of deep drawing and RTM is that the thermoplastic resin injection can be started at an arbitrary drawing depth. Therefore, Mennecart et al. [15] investigated the formability of metal-fiber-metal composites in deep drawing combined with RTM in one step, namely in situ hybridization, as shown in Fig. 5.

The thermoplastic resin is transferred through the deep drawing tool into the composite, infusing the fibers. The inlaying fibers have a significant effect on the formability of the metal blanks and therefore on the whole composite. The fibers deform the metal surface locally due to high contact forces, therefore reducing the overall formability. Therefore, metal alloys with high yield stresses are beneficial for the forming operation of those composites.

A combination of high-strength fiber-metal laminates and high-strength steel can be an interesting material for lightweight components for deep drawing applications. Specifically, battery housings for electrical vehicles offer an field of use where such materials with high specific strength and stiffness to weight ratios are needed.

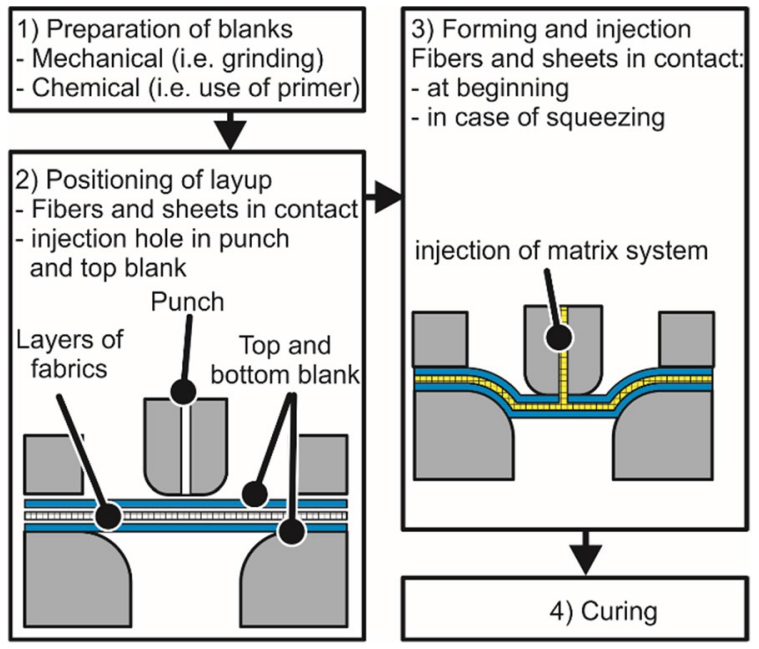

Fig. 5 Process steps of the in situ hybridization process [15]

\subsection{Hybrid Polymer/Magnesium Composites}

Completely different material properties can be advantageously combined in a hybrid polymer/magnesium composite. Magnesium has a hexagonal crystal structure which causes poor formability at room temperature so that components made of magnesium alloys are typically manufactured by casting. However, due to beneficial mechanical properties of components manufactured by metal forming, there is an increasing interest in processing magnesium wrought alloys by bulk and sheet metal forming processes [16]. Accordingly, a combined deep drawing and backmolding process has been developed by Hopmann et al. [17] for the processing of magnesium - the original process was invented by van der Aa [18]. For this process combination, the tools of a classical deep drawing process are integrated into an injection or molding machine, as shown in Fig. 6. First, the preheated magnesium alloy sheet is deep drawn. Due to this, approximately $90 \%$ of the metal component is formed. In a second forming step, the melted plastic is injected into the closed cavity. The preheating strategy of the magnesium alloy has a significant influence on its drawability. It is shown that the heating in the tool should be preferred to oven-heating due to a lower friction coefficient, resulting from this heating strategy [19].

The combination of sheet metal forming and injection molding enables a high level of functional integration in composite materials, e.g., for noise reduction purposes. Overmolding the magnesium component by a polymer die serves as protection against environmental influences and thus against corrosion [17].

\subsection{Simultaneous Deep Drawing and Cold Forging of Multi-Material Components}

Multi-material components provide an opportunity to adjust the local properties (mechanical, electrical, optical, etc.) of a component and to reduce the weight by a suitable choice of materials. A new process combination of cold forging and deep drawing for the manufacturing of multi-material components is presented by Napierala et al. [20]. The final
Fig. 6 Combined deep drawing and back molding process [19]
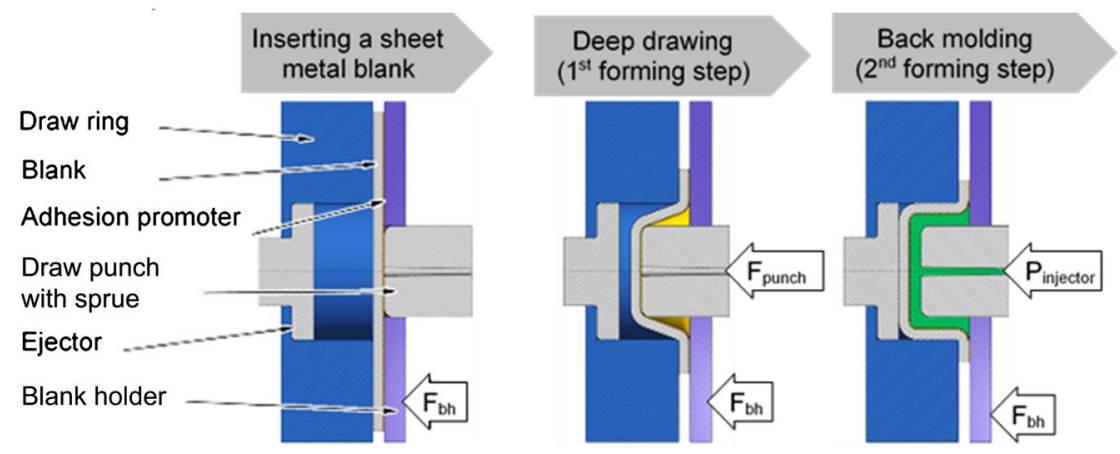
(a)

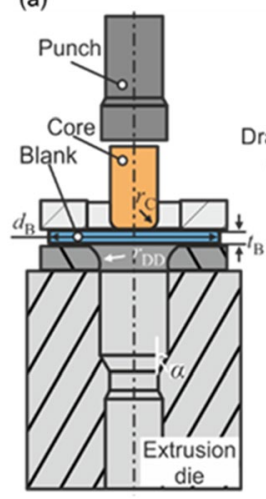

(b)

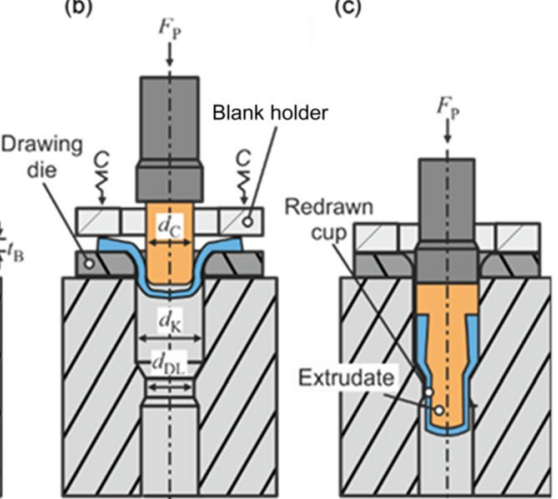

Fig. 7 Combined deep drawing and cold forging: draw forging process [20]; a initial process state; b deep drawing; c redrawing and forging

two-part component consists of a cold extruded inner core and an outer shell, which consists of a deep drawn and redrawn cup. The basic process principle of this process combination is shown in Fig. 7. In Fig. 7, $d_{\mathrm{B}}$ is the blank diameter; $t_{\mathrm{B}}$ is the blank thickness; $d_{\mathrm{C}}$ is the core diameter; $r_{\mathrm{C}}$ is the core edge radius; $r_{\mathrm{DD}}$ is the drawing die radius; $C$ is the spring constant; $d_{\mathrm{K}}$ is the container diameter; $d_{\mathrm{DL}}$ is the die land diameter; $\alpha$ is the die shoulder angle; deep drawing ratio is $\beta_{1}=d_{\mathrm{B}} / d_{\mathrm{C}}$; and nominal extrusion strain is $\varepsilon_{\mathrm{ED}}=2 \ln \left(d_{\mathrm{C}} / d_{\mathrm{DL}}\right)$. The combined draw forging tool consists of a conventional cold extrusion die extended with a deep drawing tool on top. At the beginning of the process, the core is located in the middle of the sheet surface. The sheet is placed between the deep drawing tool and the blank holder. The blank holder force can be adjusted by the blank holder springs. The process is initiated by a deep drawing process in which the core, acting as a deep drawing punch with a predefined edge radius, is pushed down by the punch, which is shown in Fig. 7b. The deep drawing process is followed by a joining process, initiated by the contact of the redrawn cup and the extrusion die shoulder, as shown in Fig. $7 \mathrm{c}$, whereby the sheet is deep drawn again and the core is cold forged. By deep drawing of a punched steel sheet and subsequent forward rod extrusion, even arbitrary shafts regions can be covered with the shell material.

For instance, components made of a steel blank and a cylindrical aluminum core can meet high requirements on the surface (hardness, corrosion resistance, electric conductivity and weight saving). The process allows the production of lightweight component such as shafts that possess different surface properties in different regions, for example, by combining various shell materials depending on the application requirements. The advantages of the process are characterized by the possibility of multi-material connection, the use of chip cores and the possibility of deep drawing with sequential backward cup extrusion. The essential connection between the connection partners in the draw forging process is based on the formation of a positive and nonpositive connection. The adhesion of the core and sleeve is based on a different elastic springback. The form fit in the axial direction is made possible by an increased thickness of the blank in the edge area. In addition, there is a form fit in the circumferential direction due to the earing of anisotropic materials. The bond strength is greater than $40 \%$ of the shear yield stress of the weakest material.

Figure 8 a shows a draw forging component which is made of a deep drawn and redrawn steel cup and an aluminum head shoulder shaft. The cup is joined over the front portion of the shaft. Such components can be used as multi-material drive shafts or in electric vehicles as connector elements with a combination of an aluminum core and a copper shell, which is presented in Fig. 8b.

\subsection{Adiabatic Blanking}

Weight reduction in automotive industry can be achieved by the use of advanced high-strength steels (AHSS). These materials demand appropriate cutting processes. Adiabatic blanking is a suitable process to process AHSS parts with excellent cutting surface quality that does not require reworking [21]. Press-hardened parts, with their extreme high strengths and surface hardnesses, are another field of application for adiabatic blanking. The characteristics of the process are high increase in temperature in a very short time in a locally limited volume of material. High local strain rates $\left(\dot{\varepsilon} \geq 10^{3} \mathrm{~s}^{-1}\right)$ and short process times $(t<2 \mathrm{~ms})$ of adiabatic blanking lead to temperature-induced softening of the material in the blanking area only. The punch travels at velocities $v \geq 3 \mathrm{~m} / \mathrm{s}$. Additionally, there is only a limited deformation of the parts, as shown in Fig. 9. As a result, the process route in comparison with the conventional blanking methods for AHSS can be reduced combined with a high cutting quality. Adiabatic shear bands (ASB) are formed during the process as a result of the high local strain rates and temperatures. Special technological properties can be achieved by the sheared surface. This includes the geometry and the mechanical properties of the shear surface. (a)

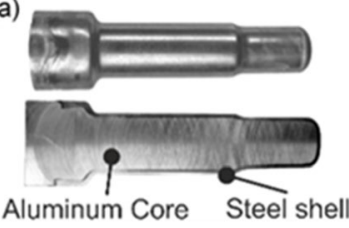

(b)

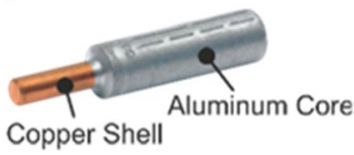

Fig. 8 Parts produced by draw forging a typical draw forged component; b Cu-Al material electrical connection 


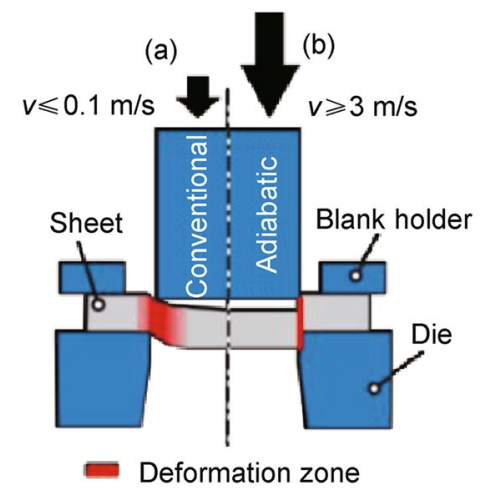

Fig. 9 Process principle: a conventional blanking process; $\mathbf{b}$ adiabatic blanking process

The adiabatic cut surface is a right-angled cutting surface that is nearly free of burrs with a fine-textured surface of fracture zone. Efficient simulation strategies and a deep process understanding are required to establish this technology in the industry as a process to cut sheet metal materials. An analytical model considering the thermomechanical material behavior is presented [22] based on material characterization of AHSS with a special focus on the strain rate and temperature-dependent material behavior. The analytical model is able to determine the local temperature increase in shear bands for high deformation rates. These data can be used to predict the final geometry of the sheared surface.

\section{Structure Lightweight Design by Forming Technology}

Structure lightweight design uses an optimized shape or topology to benefit from higher stiffnesses and structural advantages.

\subsection{Incremental Sheet-Bulk Metal Forming}

Incremental sheet-bulk metal forming offers multiple lightweight design possibilities. Complex-shaped parts and functional components with tailored properties can be produced. The production of high-strength steel parts from sheet metal is one of the key benefits of this process. Bulk forming processes are applied to sheet metal to produce near net-shaped components, by the plastic change of shape with two- and three-axial stress and strain conditions [23]. This means that a flat sheet is thickened in the out of plane direction by a forming operation and/or functional elements, like gears, attachment points or thickness changes that are integrated in the circumference of the sheet, as shown in Fig. 10. In Fig. 10, $F$ is the tool force;

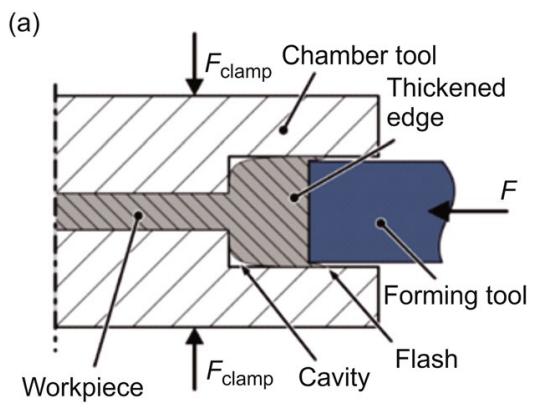

(b)

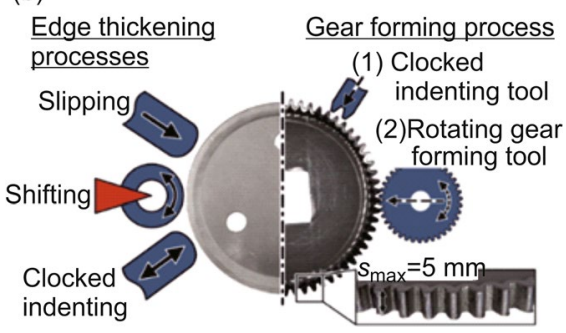

Fig. 10 Sheet-bulk metal forming processes a process principle; $\mathbf{b}$ process principles of edge thickening and gear forming by incremental sheet-bulk metal forming [24]

$F_{\text {clamp }}$ is the clamping force. The edge of the part can be thickened, and functions like gear teeth can be integrated.

An adaption of the sheet-bulk metal forming process is the incremental sheet-bulk metal forming process (iSBMF). The possibility to produce non-rotationally symmetric parts is characteristic for this process. The iSBMF process enables the efficient manufacturing of load adapted and functional components [24]. Possible applications for this process are seat adjuster or starter gears, which currently are produced conventionally, e.g., by milling, but are possible parts where iSBMF can be applied to reduce the parts weight without decrease in the parts performance, especially when using high-strength steels, as shown in Fig. 11.

The iSBMF process can be applied to produce parts to meet specific dimensions and design requirements for a specific use case, like in Fig. 11b. For this purpose, Sieczkarek et al. [23] developed a novel five-axis forming press to meet the special needs for such a process, as shown in Fig. 12.

Future research in this area will deal with the investigation of the forming behavior of hybrid parts and components. Lightweight applications for the iSBMF process are the manufacturing of gears for the drive train and seat adjuster gears. 


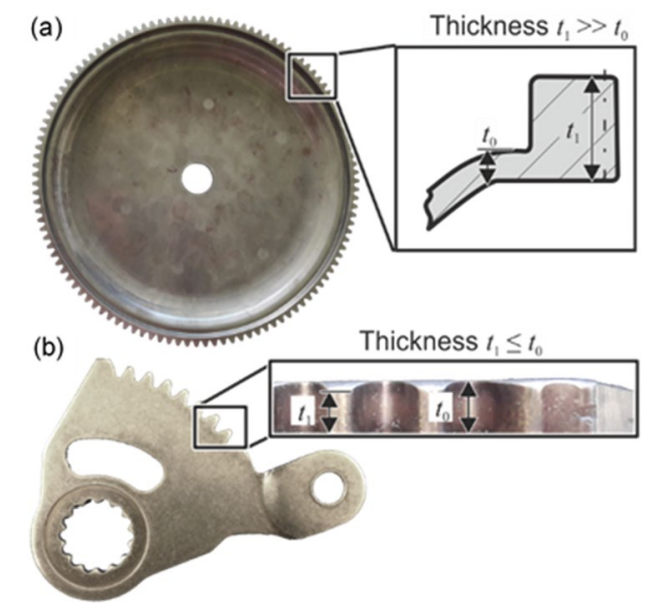

Fig. 11 Conventionally manufactured functional components: a starter gear (Winkelmann); b seat adjuster (Faurecia) [24]

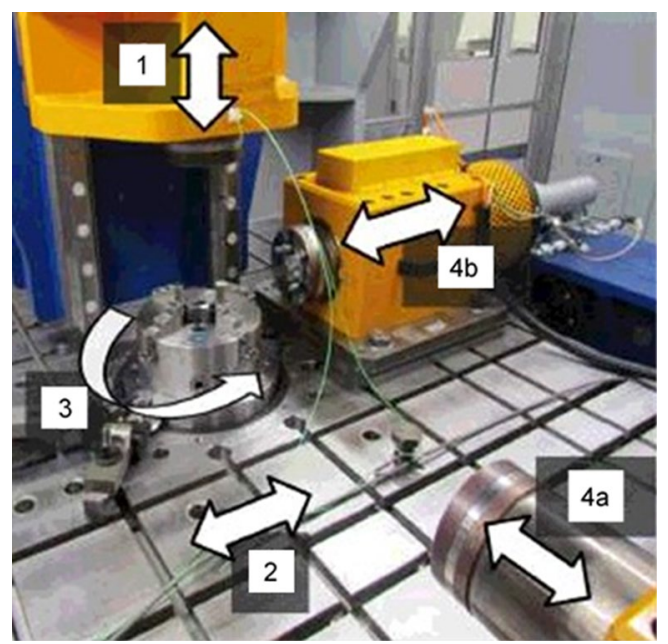

Fig. 12 Five-axis machine for the iSBMF [23]

\subsection{Joining by Forming}

Joining by forming is a process concept, which joins two materials by plastic deformation of at least one joining partner. This process is especially interesting in lightweight design since it supersedes screws, rivets or other parts in a joining operation by a form, force or material fit. Joining by plastic deformation offers the potential of improved accuracy, reliability and environmental safety and the opportunity to design new products by joining dissimilar materials [25].

\subsubsection{Magnetic Pulse Welding}

Dissimilar materials, such as hybrid driveshafts, can meet the mechanical requirements with the benefit of reduced weight. The joining of dissimilar metallic tubes to a hybrid component is challenging for conventional fusion welding processes. Therefore, magnetic pulse welding (MPW) can be utilized to join such components since it is based on the high-velocity collision between the joining partners without an additional heat source [26]. The MPW process and the flyer acceleration due to an electric discharge are shown in Fig. 13, in which $C$ is the capacitor; $R_{i}$ is the resistor. The process allows a material-bonded joint, and the process principles are as follows: A capacitor bank provides electrical energy that can be discharged within several microseconds and induces a secondary current through the magnetic field in the electroconductive flyer. The flyer is accelerated in radial direction by the Lorentz forces that act between the coil and the flyer. The flyer collides with the fixed inner part at a high velocity and creates a high pressure at the joining partners interface. As a result, a so-called jet is formed which consists of surface particles of both joining partners [26], accompanied by a characteristic light emission to monitor and control the MPW process [27].

Utilizing the MPW process, Lueg-Althoff et al. [26] presented successful experimental research to join dissimilar metallic tubes (aluminum flyers to steel parents, aluminum flyers to copper parents) with reduced wall thicknesses. A possible application is the production of driveshafts that consist of different materials [28]. With this approach, a bi-metallic stub shaft was produced to an aluminum-steel drive shaft, as shown in Fig. 14.

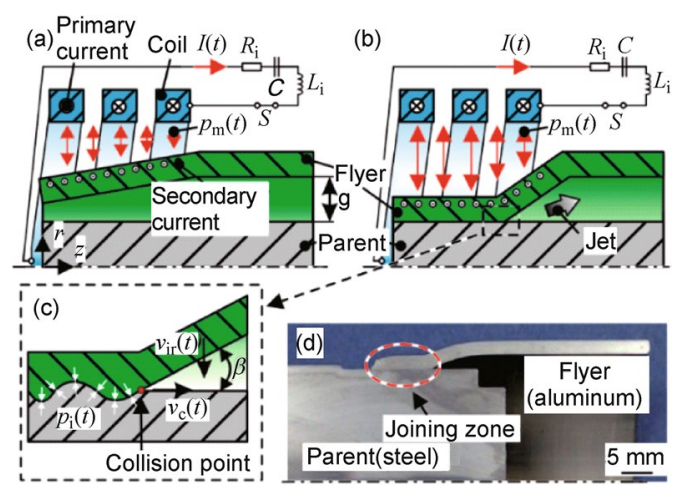

Fig. 13 MPW process by electromagnetic compression: a Electromagnetic acceleration of the flyer; $\mathbf{b}$ collision between flyer and parent; c processes at the collision zone; d example of an aluminumsteel joint [26] 


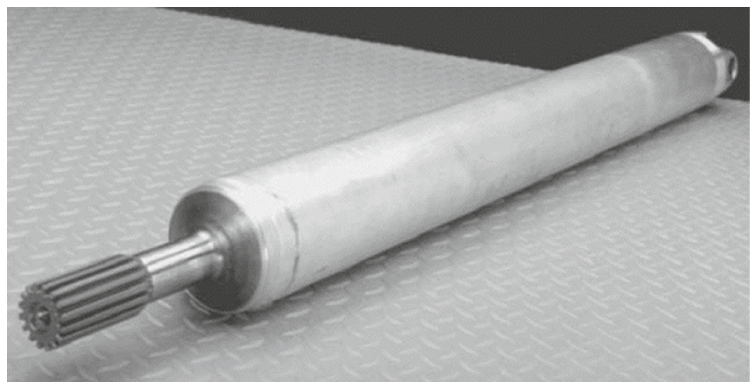

Fig. 14 Spicer bi-metallic stub shaft produced by magnetic pulse welding of aluminum and steel [28]

\subsubsection{Joining by Die-less Hydroforming}

To produce joints without the need of an external heat source, joining by die-less hydroforming (DHF) can be used. Müller et al. [29] presented a joining process to produce overlap joints by means of hydraulic expansion. The principle lies in the difference of the elastic recovery of the two joining partners due to a deformation and therefore a remaining radial contact pressure in their contact area. This offers great potential for joining parts in lightweight applications. Rotational symmetric as well as non-rotational symmetric [29] profiles can be joined. Interference-fit joints and form-fit joints can be produced. Interference-fit joints rely on the friction between the overlap of the joining partners. Form-fit joints generate an undercut between the inner and the outer joining partners, as shown in Fig. 15. The formfit joint has great advantages by the produced undercut for connections under torque load. The bearable load already increases significantly with minimal overlap of some tenths of a millimeter [30].

For the manufacturing of lightweight structures, DHF is a great alternative to thermal-based joining processes.

Next to the production of driveshafts, another application is the production of lightweight framework structures. Marré et al. [31] investigated the connection of several tubular parts by a node to create an assembly inspired by the lightweight frame structure for the BMW C1E motorcycle, as shown in Fig. 16.

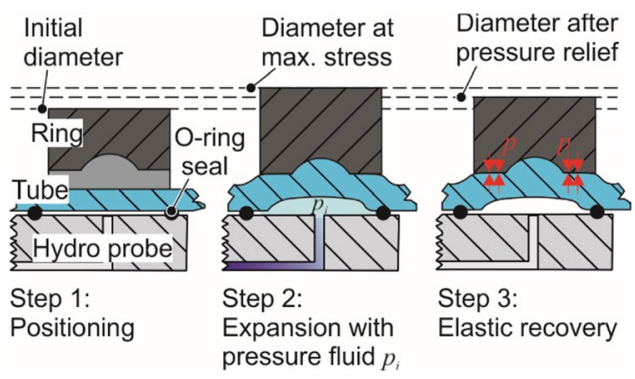

Fig. 15 Process sequence for joining by die-less hydroforming [29]

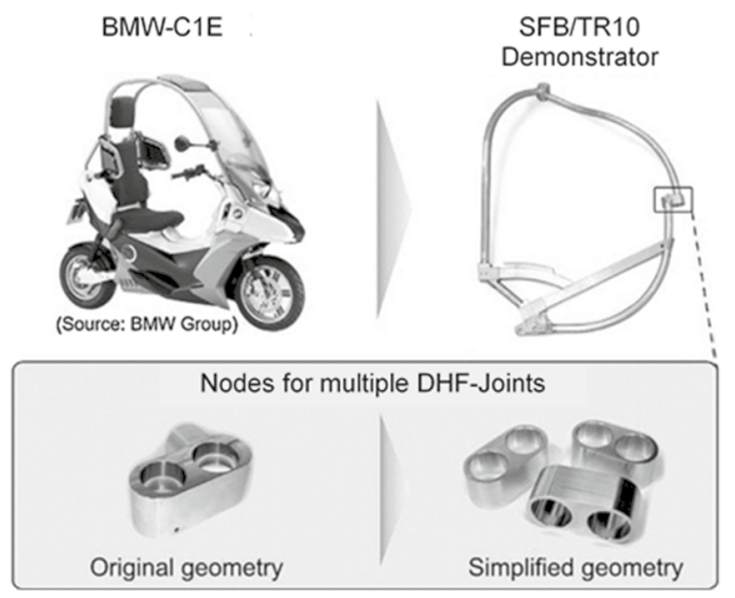

Fig. 16 Lightweight frame structure inspired by the BMW C1E motorcycle [31]

\subsection{Profiles/Tubes with Variable Cross Section}

The adjustment of tube diameters and bending curvatures can be used to produce load-adjusted structures with variable tube topology and the possibility to form high-strength materials, which is a current trend in lightweight design [32]. Due to the increased tendency of springback, highstrength materials are challenging to bend [33].

\subsubsection{Incremental Tube Forming}

To overcome and reduce problems related to springback of high-strength materials, the incremental tube forming (ITF) process was invented [34]. ITF combines the technology of incremental tube spinning and continuous tube bending, where both processes take place simultaneously. The process offers the possibility to produce tubes with variable diameter and freely definable bending curvatures, as shown in Fig. 17, in which $R_{\mathrm{L}}$ is the bending radius.

The initial tube is fed through rotating spinning rolls to set the wanted tube diameter and the magnitude for the stress superposition. At the same time, the bending tool superposes an additional bending moment to give the tube the desired shape, as shown in Fig. 17a. Thus, tubes can be bent from high-strength steels DP 800 and DP 1000, respectively, as shown in Fig. 17b. The stress superposition causes a significant reduction in the bending moment, compared to conventional tube bending processes [36]. The possibility to almost freely form high-strength materials can be used to produce parts like anti-roll bars for automotive applications.

Another application for the ITF process is the manufacturing of titanium tubes to run at elevated temperatures [37]. Titanium tubes offer a high strength-to-weight ratio and corrosion resistance which makes them ideal for lightweight applications, especially in corrosive environments. 


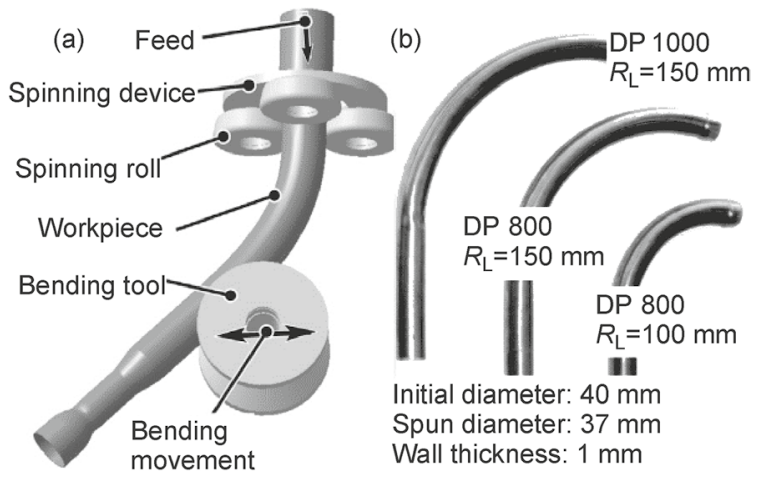

Fig. 17 Incremental tube forming (ITF): a process principle; b application [35]

Unfortunately, titanium suffers from its low formability in combination with high strength, so it is challenging to form in a defined bending operation. The adaption of the ITF process with an additional inductor can be used to heat up the titanium tube for higher ductility. In combination with the reduced bending forces by stress superposition, the device offers the potential to bend titanium tubes to complex shapes with good dimensional stability [37].

\subsubsection{Incremental Profile Forming}

The production of profiles with variable cross sections, the incremental profile forming (IPF) process, was developed. The process allows the production of tubes and profiles with variable cross-sectional designs along the center line of a tube or profile [38]. The process combines high process flexibility with high workpiece complexity. Grzancic et al. [38] developed a new machine concept and a prototype which allows the processing of thin-walled tubes and profiles with diameters up to $80 \mathrm{~mm}$, as shown in Fig. 18. The highly flexible forming process can be considered as incremental, since it preceeds in several steps.
Figure 18a shows the general process principle that was first described by Staupendahl et al. [39], in which $z_{\mathrm{p}}$ is the profile feed; $r_{\mathrm{st}, 1}$ and $r_{\mathrm{st}, 2}$ are the styluses feed; $\alpha$ is the rotation. A varying number of styluses are arranged circularly around the profile axis which can move independently from one another into the profile. The initial cross section can be diverse, e.g., quadric, circular, rectangular, etc. The forming of the tube takes place while the profile is fed in axial direction and the styluses penetrate the workpiece in radial directions [38]. To increase the flexibility, either the workpiece or the styluses can rotate-both concepts have the same effect on the forming operation. The process can apply a symmetric or an asymmetric shape into the workpiece, depending on the tooling setup, as shown in Fig. 18b. Through the flexibility of this process, multiple combinations of tools, tool geometries, process setups and profile shapes including an enormous amount of final workpiece shapes can be realized, as shown in Fig. 18c. With special tool kinematics, profile forming from the inside is also possible. Such profiles can be utilized in lightweight applications to meet specific design requirements or increase stiffnesses in multiple directions by a change of the profiles topology.

\subsection{Damage-Controlled Forming Processes}

Damage in metal-formed parts is not a failure, but a product property that decreases the performance of components and needs to be considered in the dimension of products [40]. Due to the appearance and evolution of voids, damage is evolving in a part during a specific forming operation and reduces its load bearing capacity [40]. It is important to understand this when dealing with ductile damage, failure and product properties, especially when dealing with lightweighting in metal-formed parts. As a result of the known damage state inside the part, it is possible to properly rate a construction for its specific use case. The Collaborative Research Centre TR188 "Damage Controlled
Fig. 18 Incremental profile forming (IPF) a process kinematics; $\mathbf{b}$ possible setups of the machine; c sample workpieces from different machine setups $[38,39]$ (a)

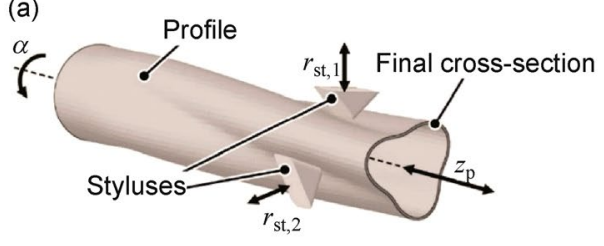

(b)

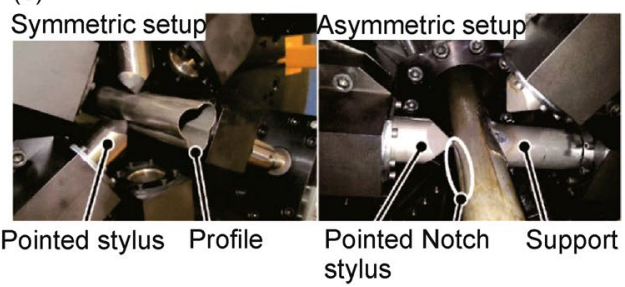

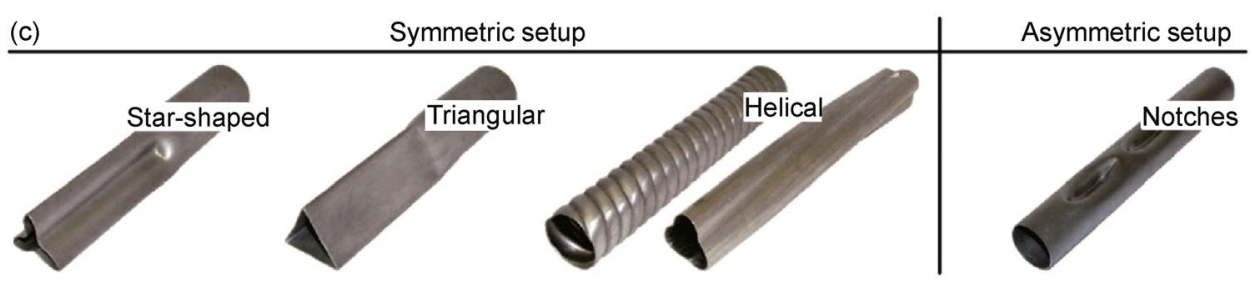


Forming Processes" has the aim to introduce a paradigm shift in forming industry. Instead of only considering the manufacturability, forming-induced product properties, e.g., damage, should be considered in the process and product design cycle - a reorientation from formability to usability is initiated in the TR188 research center.

\subsubsection{Damage in Cold Forging}

Product properties are dependent on the damage evolution introduced by the forming operation. Reducing and knowing the amount of damage help to further increase the lightweighting potential. Tekkaya et al. [41] experimentally investigated the damage evolution and fatigue strength of cold-forged parts and of parts produced by air bending. The increase in the extrusion ratio leads to a lower damage and increased fatigue strength, whereas in an air bending operation the damage can be influenced by process variations such as stress superposition. Cold-forged parts with different axial stress values during the extrusion process exhibit different damage evolutions, as shown in Fig. 19a. Modeling parameters are as follows: cone angle $2 \alpha=90^{\circ}$; friction coefficient $m=0.08$ (Coulomb); elastic die (55NiCrMoV6); elastic-plastic workpiece (16MnCr5); initial rod diameter $d_{0}$.

The process results in lower damage for higher strains (Fig. 19b), whereas the specimen exhibiting the lower strain has a higher axial tensile stress (Fig. 19a). As a result, the fatigue strength of the specimen who were strained to a lower value $(\varepsilon=0.5)$ shows the lower fatigue strength (Fig. 19b). Thus, a larger extrusion strain leads to less damage and increased fatigue strength [41].

\subsubsection{Damage in Bending}

Air bending experiments with and without an elastomer cushion were performed, as shown in Fig. 20a, b. Parameters for simulation are as follows: friction coefficient $m=0.3$; die and tool are assumed as rigid bodies; sheet metal is modeled with elastic-plastic material and isotropic hardening (DP1180); elastomer cushion is modeled with the Mooney-Rivlin model. Bending with the superimposed compressive stresses of the elastomer cushion decreases the stress triaxiality $\eta=\sigma_{\mathrm{h}} / \sigma_{\mathrm{V}}$ (hydrostatic stress divided by von Mises stress) by $18 \%$, as shown in Fig. 20c, d. Triaxiality is typically defined between -1 and 1 . A negative triaxiality results in void decrease and shrinkage, while a positive triaxiality results in void growth and therefore increased damage values. As a consequence, the damage introduced by the elastomer cushion should be lower which could be proved by micrographs of the bending specimens after the bending operations. As a result, the fatigue life of the specimens formed into the elastomer cushion is higher by approximately $10 \%$.
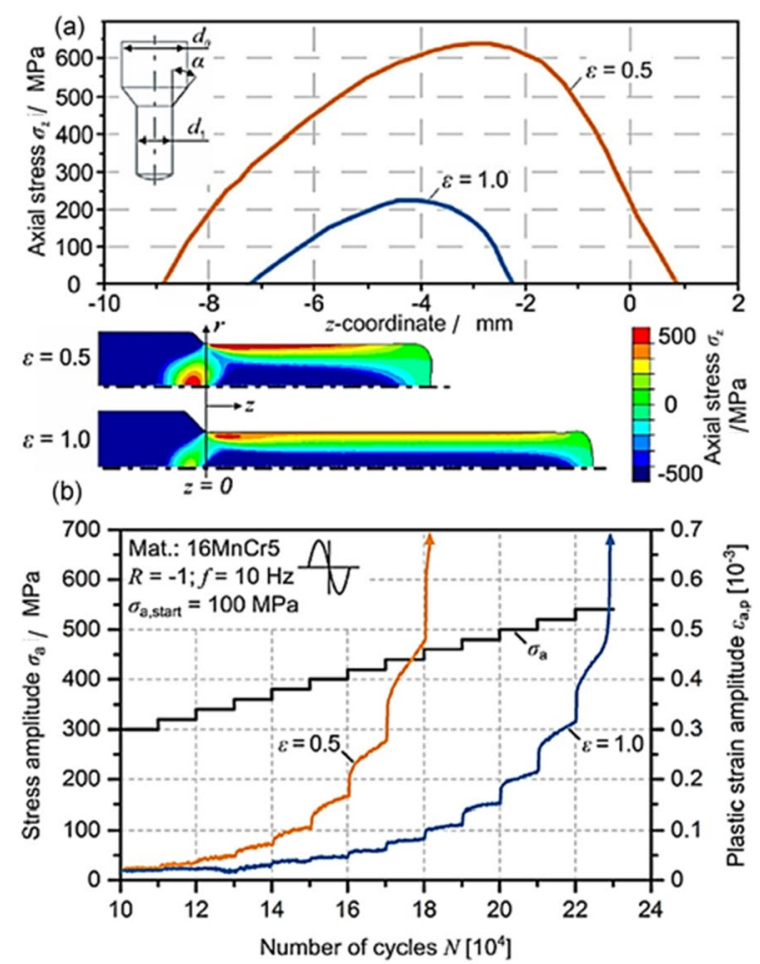

Fig. 19 Damage evolution in forward rod extrusion: a forward rod extrusion and the influence of the area reduction on the axial tensile stresses; b non-heat-treated samples after cold forging and the influence of the strain on the fatigue strength [41]

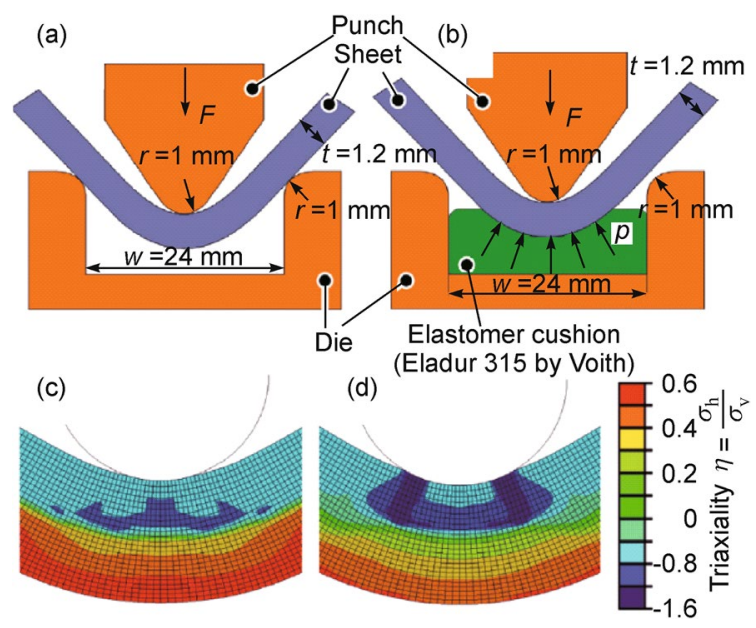

Fig. 20 Air bending process: a simple air bending; $\mathbf{b}$ bending into an elastomer cushion. Triaxiality for $\mathbf{c}$ simple air bending and $\mathbf{d}$ bending into an elastomer cushion [41]

A new process was developed by Meya et al. [42]. The novel sheet metal bending process was developed to adjust the load path to control the damage evolution by a superposition of radial stresses during bending — namely radial 


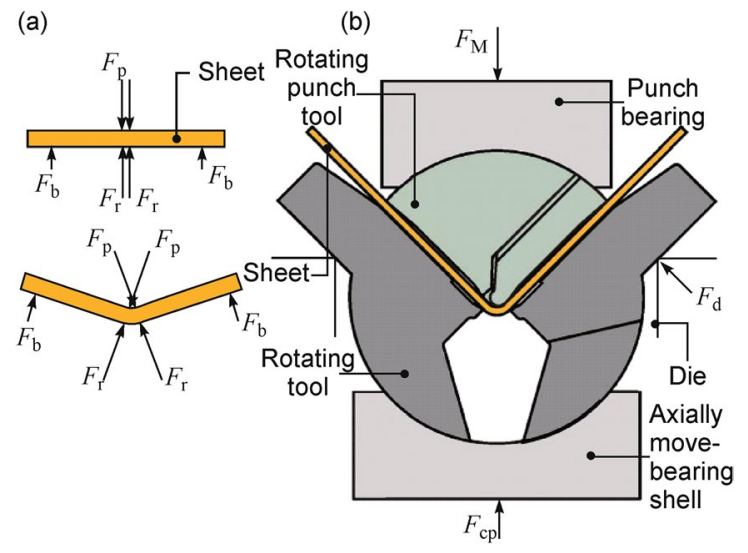

Fig. 21 Principle of RSS bending: a at initial state (upper figure) and during bending (lower figure) and $\mathbf{b}$ technological implementation [43]

stress superposed (RSS) bending, as shown in Fig. 21 [43]. Through the changed stress state of the new process, a reduction in triaxiality can be achieved which inhibits the initiation and growth of voids. By the control of the damage evolution during the bending process, a better product performance, e.g., in terms of impact absorbance, can be achieved.

\section{System Lightweight Design}

System lightweight design means the integration of multiple parts or functions into a single part or system to reduce the weight of an assembly. The aforementioned lightweight design strategies, namely material lightweight design in Introduction and structure lightweight design in Sect. 3, are subsets of the system lightweight design. Typically those are combined into a system to produce a light assembly-usually lighter than the preliminary development stage (refer to the true lightweight degree [1]).

In the context of forming technology, the production of systems through forming is hard to achieve, since forming technology is used to manufacture components or process new materials.

\subsection{Combination of Additive Manufacturing and Forming}

A new concept to produce light parts is the combination of additive manufacturing (AM) and forming technology. The additive manufacturing of highly complex parts with integrated functions, like cooling channels, sensors or connector elements, currently suffers from low process efficiency caused by low manufacturing times and an often inefficient usage of build chamber volumes during the AM process.

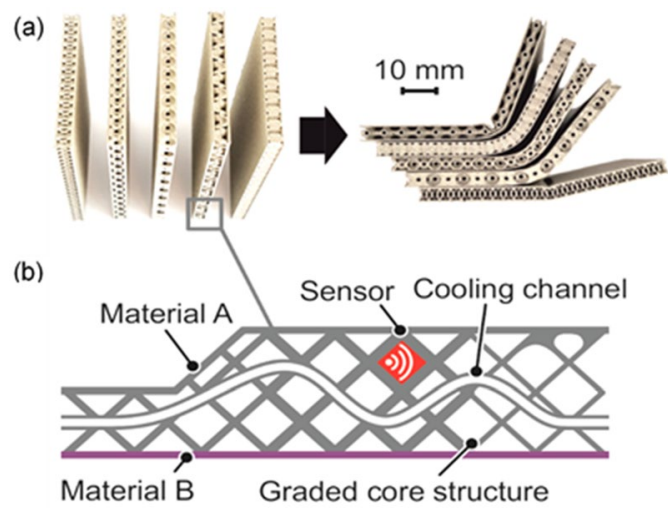

Fig. 22 Combination of additive manufacturing and forming: a additively manufactured sandwich sheet bent until failure; b schematically possible functional integrations through additive manufacturing (partly taken from [44])

To overcome this problem, Rosenthal et al. [44] proposed a new process combination that utilizes the benefits of additive manufacturing (production of complex sheets) and forming technology (leads to strain hardening). The subsequent forming of additively manufactured parts enables the production of flat sheets with a core structure and integrated functions that are stacked in the build chamber and nearly uses the whole available build chamber volume, as shown in Fig. 22.

In the subsequent forming operation, the final geometry is set, with the benefit of an increased strength through work hardening. Therefore, the weight of the final part can be reduced compared to a part that is additively produced in final geometry. The usability of this approach has been shown by a bending operation of solid additively manufactured sheets [45].

Another approach which combines additive manufacturing and forming technology is the combination of two point incremental forming (TPIF) and laser powder deposition. A 3-dimensional part is produced by TPIF, and in the same setup functional elements, like ribs, fins or attachment points, are welded on top of the sheet [46]. A great benefit of this process combination is the efficiency of both processes and the flexibility of them that do not need a specific tool to produce different parts. Theoretically, every produced part can be individualized.

\subsection{Shape Memory Alloy Metal Matrix Composites}

Shape memory metal matrix composites are a novel approach to producing components on the level of system lightweight design. Dahnke et al. [47] presented a new actuator concept based on integrated nickel-titanium (NiTi) wires within an aluminum matrix, which is produced in a continuous composite extrusion process, as shown in Fig. 23, in which $M_{\mathrm{f}}$ is the martensite start temperature; $A_{\mathrm{f}}$ is the 


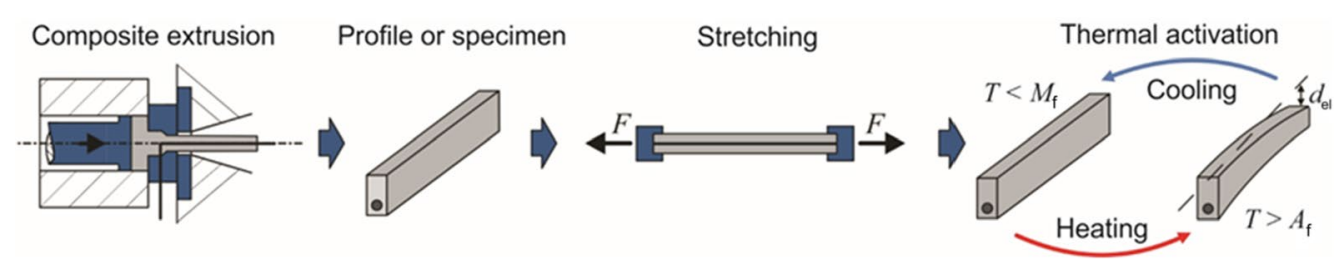

Fig. 23 Process chain for the manufacturing of bending actuators by means of composite extrusion [47]

austenite start temperature. An eccentric positioning of the NiTi wires and an induced prestraining cause a bending moment in the composite when the temperature is increased to a critical value. As a result, an elastic or elastic-plastic deformation of the profile can be triggered, and therefore, such profiles have the ability for a repeatable change of shape which can be controlled by the ambient temperature.

The above presented concepts for system lightweight design are just three examples of how functions and processes can be combined to produce light parts. The majority of parts that are produced through forming technologies are on the component level and are combined to a system in a later stage of the production chain.

\section{Conclusions}

The need to promote lightweight design strategies by new and innovative processes is motivated. A subdivision into system, structure and material lightweight design has been described. The system lightweight design is to be seen as a holistic concept, where a complete automobile is considered. Structural lightweight design considers the component level and has to be regarded as a subset of the lightweight system design. Material lightweight design is the fundamental level and is a subset of structural lightweight design. The structure of this paper follows this classification and shows innovative processes at each level, creating new potential in lightweight design. Possible applications in the automotive sector are visualized in Figs. 1 and 2, motivated by different examples.

In material lightweight design, weight reduction is reached by using lighter materials with specific properties (e.g., strength). Examples of this are high-strength steels, aluminum or magnesium alloys and composite materials. The processes, which are presented in this paper, deal with thermal supported processes such as press hardening, whereby complex components with extremely high-strength properties can be produced through simultaneous forming and heat treatment. Also, the post-treatment process for hardened steels is shown in the form of adiabatic shear cutting. This process allows-due to the high local deformation rate-a significant and concentrated increase in the temperature and so a decrease in the required cutting force.
Furthermore, metal-polymer materials are presented, where hybrid materials can be produced through a targeted and process-integrated combination of lightweight metals and polymers. On the bulk forming side, a process combination of extrusion and deep drawing is presented, which enables the production of hybrid shafts.

In structure lightweight design, among other processes, joining by forming is explained. Therefore, either hydraulic or electromagnetic mechanisms are used to combine different metal components. Furthermore, the process of sheet-bulk metal forming is presented, which can be used to produce functional components with tailored properties regarding the material distribution. The production of components with tailored properties is also described for the area of profile forming. Two processes are shown in detail which allow the incremental bending and/or shaping of the cross section of tubes. As the last part of structural lightweight design, an insight into the topic of damage is given. It shows how the damage evolution of a workpiece can be influenced during manufacturing by means of suitable process parameters and/or stress superposition. As a result, they have a lower void density and better performance. Due to the fact that damage is known, fewer safety reserves are necessary and components can be dimensioned lighter.

The system lightweight design in this paper is represented by forming process approaches, which are used to manufacture components with functional integration. On the one hand, additively manufactured sandwich sheets with complex core structures are used for forming operations. Through the manufacturing process of the semi-finished product, the integration of a variety of functions such as cooling channels is possible. The subsequent forming operation is led by work hardening, when the benefits of AM and forming technology are combined in this process. Furthermore, a composite material out of shape memory alloy wires in a metal matrix is presented. These parts allow an elastic or elastic-plastic deformation of the profile triggered by the process by the ambient temperature.

An assessment or comparison of current lightweight design approaches is difficult to carry out at the current time. A simple consideration of the weight saving is not expedient for this purpose. The integration of the functional level is required here, whereby the requirements 
for comparable components are also taken into account. Hahn et al. [1] present a suitable approach at the component level. In the future, this analytical description should also be expanded at the strength level. This would enable a quantitative comparison of different components and a chain of several successive lightweight design approaches.

\begin{abstract}
Acknowledgement Open Access funding provided by Projekt DEAL. The authors thank the German Research Foundation (DFG) for the financial support of the ongoing projects: Collaborative Research Centre TRR 188 "Damage Controlled Forming Processes" (Project number 278868966-TRR 188); Forming of additively manufactured sandwich sheets with optimized core structures (Project number 317137194); Process combination of single point incremental forming and laser powder deposition for the manufacturing of lightweight components (Project number 385276922); Joining by die-less hydroforming with outer pressurization (Project number 350070123); Process combination of combined deep drawing and cold forging (Project number 289596321); Kinematic profile bending with locally heated cross section (Project number 408302329); Product property controlled multistage hot sheet metal forming (Project number 424334660); TRR 73: Manufacturing of Complex Functional Components with Variants by Using a New Sheet Metal Forming Process - Sheet-Bulk Metal Forming (Project number 68237143).
\end{abstract}

\section{Compliance with Ethical Standards}

Conflict of interest On behalf of all the authors, the corresponding author states that there is no conflict of interest.

Open Access This article is licensed under a Creative Commons Attribution 4.0 International License, which permits use, sharing, adaptation, distribution and reproduction in any medium or format, as long as you give appropriate credit to the original author(s) and the source, provide a link to the Creative Commons licence, and indicate if changes were made. The images or other third party material in this article are included in the article's Creative Commons licence, unless indicated otherwise in a credit line to the material. If material is not included in the article's Creative Commons licence and your intended use is not permitted by statutory regulation or exceeds the permitted use, you will need to obtain permission directly from the copyright holder. To view a copy of this licence, visit http://creativecommons.org/licenses/by/4.0/.

\section{References}

1. Hahn, M., Gies, S., Tekkaya, A.E.: Light enough or go lighter? Mater. Des. (2019). https://doi.org/10.1016/j.matde s. 2018.107545

2. Justen, R., Schöneburg, R.: Crash safety of hybrid- and battery electric vehicles. In: Proceedings of the 22nd International Technical Conference on the Enhanced Safety of Vehicles, NHTSA, Washington, 13-16 June 2011

3. Tekkaya, A.E., Khalifa, N.B., Grzancic, G., et al.: Forming of lightweight metal components: need for new technologies. Procedia Eng. (2014). https://doi.org/10.1016/j.proeng.2014.09.125

4. Tekkaya, A.E., Allwood, J.M., Bariani, P.F., et al.: Metal forming beyond shaping predicting and setting product properties. CIRP Ann. (2015). https://doi.org/10.1016/j.cirp.2015.05.001

5. Bartos, R.: Stahl für nachhaltige mobilitat (engl. Steel for sustainable mobility). https://www.stahl-online.de/index.php/\%20 themen/stahlanwendung/mobilitaet/. Accessed 17 February 2020

6. Neugebauer, R., Altan, T., Geiger, M., et al.: Sheet metal forming at elevated temperatures. CIRP Annals (2006). https://doi. org/10.1016/j.cirp.2006.10.008

7. Chen, H., Güner, A., Khalifa, N.B., et al.: Granular media-based tube press hardening. J. Mater. Process. Technol. 228, 145-159 (2016). https://doi.org/10.1016/j.jmatprotec.2015.03.028

8. Chen, H., Hess, S., Haeberle, J., et al.: Enhanced granular medium-based tube and hollow profile press hardening. CIRP Annals. 65, 273-276 (2016). https://doi.org/10.1016/j. cirp.2016.04.010

9. Chen, H., Hess, S., Güner, A., et al.: Active and passive granular media-based tube press hardening. Paper presented at 10th Erlanger Workshop Warmblechumformung, Universität Erlangen-Nürnberg, Nürnberg, 3 November 2014

10. Löbbe, C., Tekkaya, A.E.: Mechanisms for controlling springback and strength in heat-assisted sheet forming. CIRP Annals. 67, 273-276 (2018). https://doi.org/10.1016/j.cirp.2018.04.013

11. Becker, C., Hermes, M., Löbbe, C., et al.: Verfahren und Vorrichtung zur Beeinflussung des Umformungsverhaltens, insbesondere der Rückfederung bei Umformung von Werkstücken in getakteten Umform- und Bearbeitungsstationen, insbesondere beim Biegen in Folgeverbundwerkzeugen. Patent DE102014001872B4, 19. October 2019

12. Kolleck, R., Veit, R., Merklein, M., et al.: Investigation on induction heating for hot stamping of boron alloyed steels. CIRP Annals. 58, 275-278 (2009). https://doi.org/10.1016/j. cirp.2009.03.090

13. Miller, W.K.: Metal-Plastic laminates for vehicle weight reduction. In: 1980 Automotive Engineering Congress and Exposition. SAE International, Warrendale, 1 February (1980). https ://doi.org/10.4271/800077

14. Mennecart, T., Werner, H., Ben Khalifa, et al.: Developments and analyses of alternative processes for the manufacturing of fiber metal laminates. In: Volume 2: Materials; Joint MSECNAMRC-Manufacturing USA, ASME 2018 13th International Manufacturing Science and Engineering Conference, College Station, Texas, USA, 18-22 June 2018. https://doi.org/10.1115/ MSEC2018-6447

15. Mennecart, T., Gies, S., Khalifa, Ben, et al.: Analysis of the influence of fibers on the formability of metal blanks in manufacturing processes for fiber metal laminates. J. Manuf. Mater. Process. (2019). https://doi.org/10.3390/jmmp3010002

16. Dröder, K.G.: Untersuchungen zum Umformen von Feinblechen aus Magnesiumknetlegierungen. Dissertation, Leibniz Universität Hannover (1999)

17. Hopmann, C., Wurzbacher, S., Tekkaya, E., et al.: Deep-drawing and backmolding process for plastic-magnesium hybrids. Lightweight Design Worldwide 11, 58-63 (2018). https://doi. org/10.1007/s41777-018-0015-y

18. Van der Aa, H., Verdier, A., Wolf, H. de: Method and device for producing a composite product, and composite product produced therewith. Patent WO 03/057446, 17 July 2003

19. Joghan, H. D., Hahn, M., Traphöner, H., et al.: Influence of the preheating strategy on the deep drawing of extruded magnesium alloy ME20 sheets. In: IOP Conf. Ser.: Mater. Sci. Eng. (2019). https://doi.org/10.1088/1757-899X/651/1/012067

20. Napierala, O., Dahnke, C., Tekkaya, A.E.: Simultaneous deep drawing and cold forging of multi-material components: drawforging. CIRP Annals. 68(1), 269-272 (2019). https://doi. org/10.1016/j.cirp.2019.03.001

21. Bai, Y., Dodd, B.: Adiabatic Shear Localization: Occurrence, Theories and Applications. Pergamon Press, Paris (1992)

22. Winter, S., Schmitz, F., Clausmeyer, T., et al.: High temperature and dynamic testing of AHSS for an analytical description of the 
adiabatic cutting process. In: IOP Conf. Ser.: Mater. Sci. Eng. (2017). https://doi.org/10.1088/1757-899X/181/1/012026

23. Sieczkarek, P., Kwiatkowski, L., Ben Khalifa, N., et al.: Novel five-axis forming press for the incremental sheet-bulk metal forming. Key Eng. Mater. 554-557, 1478-1483 (2013). https://doi. org/10.4028/www.scientific.net/KEM.554-557.1478

24. Wernicke, S., Sieczkarek, P., Gies, S., et al.: Properties of components with incrementally formed gears. Metals. 9(5), 515 (2019)

25. Mori, K.-I., Bay, N., Fratini, L., et al.: Joining by plastic deformation. CIRP Annals 62(2), 673-694 (2013). https://doi. org/10.1016/j.cirp.2013.05.004

26. Lueg-Althoff, J., Bellmann, J., Hahn, M., et al.: Joining dissimilar thin-walled tubes by magnetic pulse welding. J. Mater. Process (2020). https://doi.org/10.1016/j.jmatprotec.2019.116562

27. Bellmann, J., Beyer, E., Lueg-Althoff, J., et al.: Measurement of collision conditions in magnetic pulse welding processes. J. Phys. 7(4), 1-10 (2017). https://doi.org/10.17265 /2159-5348/2017.04.001

28. Kochan, A.: Magnetic pulse welding shows potential for automotive applications. Assembly Autom. 20(2), 129-132 (2000). https ://doi.org/10.1108/01445150010321742

29. Müller, M., Gies, S., Tekkaya, A.E.: Joining by die-less hydroforming of profiles with oval cross section. KEM. 767, 405-412 (2018). https://doi.org/10.4028/www.scientific.net/KEM.767.405

30. Krips, H., Podhorsky, M.: Hydraulisches aufweiten - ein neues verfahren zur befestigung von rohren. VGB Kraftwerkstechnik. 56, 456-464 (1976)

31. Marré, M., Gies, S., Maevus, F., et al.: Joining of lightweight frame structures by die-less hydroforming. Int. J. Mater. Form. 3, 1031-1034 (2010). https://doi.org/10.1007/s12289-010-0946-2

32. Kleiner, M., Geiger, M., Klaus, A.: Manufacturing of lightweight components by metal forming. CIRP Annals 52(2), 521-542 (2003). https://doi.org/10.1016/S0007-8506(07)60202-9

33. Wagoner, R.H., Lim, H., Lee, M.-G.: Advanced issues in springback. Int. J. Plast. 45, 3-10 (2013). https://doi.org/10.1016/j.ijpla s.2012.08.006

34. Hermes, M., Kurze, B., Tekkaya, A.E.: Verfahren und Vorrichtung zur Umformung eines Stangenmaterials (Method and device for forming a bar stock). Patent EP000002203264B1, 28 September 2007

35. Becker, C., Tekkaya, A.E., Kleiner, M.: Fundamentals of the incremental tube forming process. CIRP Annals 63(1), 253-256 (2014). https://doi.org/10.1016/j.cirp.2014.03.009

36. Hermes, M., Staupendahl, D., Becker, C., et al.: Innovative machine concepts for 3D bending of tubes and profiles. Key Eng.
Mater. 473, 37-42 (2011). https://doi.org/10.4028/www.scientific .net/KEM.473.37

37. Gallus, S., Meya, R., Seibt, M., et al.: Manufacturing of tailored tubes at elevated temperatures by heat assisted incremental forming. Paper presented at the 2nd Conference \& Exhibition on Light Materials, Bremen, 09 June (2017)

38. Grzancic, G., Becker, C., Hermes, M., et al.: Innovative machine design for incremental profile forming. Key Eng. Mater. 622413, 413-419 (2014). https://doi.org/10.4028/www.scientific.net/ KEM.622-623.413

39. Staupendahl, D., Becker, C., Weinrich, A., et al.: Innovative Umformverfahren für Rohre, Profile und Bleche aus modernen Stahlwerkstoffen. Stahl und Eisen 132(8), 47-54 (2012)

40. Hering, O., Tekkaya, A.E.: Damage-induced performance variations of cold forged parts. J. Mater. Process. 279, 116556 (2020). https://doi.org/10.1016/j.jmatprotec.2019.116556

41. Tekkaya, A.E., Ben Khalifa, N., Hering, O., et al.: Forminginduced damage and its effects on product properties. CIRP Annals 66(1), 281-284 (2017). https://doi.org/10.1016/j. cirp.2017.04.113

42. Meya, R., Löbbe, C., Tekkaya, A.E.: Device and method of bending metal sheet during simultaneous pressure tension superimposition. Patent DE102017006218, 26 June 2017

43. Meya, R., Löbbe, C., Tekkaya, A.E.: Stress state control by a novel bending process and its effect on damage and product performance. In: Proceedings of the 13th Manufacturing Science and Engineering Conference (2018)

44. Rosenthal, S., Platt, S., Hölker-Jäger, R., et al.: Forming properties of additively manufactured monolithic Hastelloy X sheets. Mater. Sci. Eng. A. 753, 300-316 (2019). https://doi.org/10.1016/j. msea.2019.03.035

45. Rosenthal, S., Hahn, M., Tekkaya, A.E.: Simulation approach for three-point plastic bending of additively manufactured Hastelloy X sheets. Procedia Manuf. 34, 475-481 (2019). https://doi. org/10.1016/j.promfg.2019.06.201

46. Hölker, R., Ben Khalifa, N., Tekkaya, A.E.: Verfahren und Vorrichtung zur kombinierten Herstellung von Bauteilen mittels inkrementeller Blechumformung und additiver Verfahren in einer Aufspannung. Patent DE102014014202A1, 24 March (2016)

47. Dahnke, C., Shapovalov, A., Tekkaya, A.E.: Thermally activated lightweight actuator based on hot extruded shape memory metal matrix composites (SMA-MMC). Procedia Eng. 207, 1511-1516 (2017). https://doi.org/10.1016/j.proeng.2017.10.1083 